\title{
Research of Magnetotydrodynamics in a Free-Burning
}

\author{
Yi Wang, Xiong Chen, Hao Chen and ZhengZhi Du \\ Nanjing University of Sciecce\&Technology, China \\ Jiangsu Power Equipment co., LTD, China
}

Keywords: free-burning arc, magnetic vector potential, coupling calculation of flow field and cathode melting, electromagnetic field, anode vapor.

\begin{abstract}
By means of magneto hydrodynamic modelling based on magnetic vector potential and UDF programming, a numerical simulation is conducted for the heat transfer and flow process in the free-burning arc, which enables the coupling calculation of flow field and electromagnetic field. In consideration of the catholic tip melting and the copper vapor generated by anodic copper sheet evaporation during the arc burning process, the interaction between arc and vapor is analyzed and the influence of electrode melting on the flow and heat transfer of plasma arc is therefore fully understood, making the calculation model better represent the actual situation.
\end{abstract}

\section{Introduction}

Plasma arc technology has been widely applied in metallurgy, spray coating, cutting and organic waste disposal sectors. During the arc burning process, the conductive thermal plasma can be formed. Taking plasma as the continuum, numerical simulation of arc is carried out with the aid of magnetohydrodynamic model. Hsu ${ }^{[1]}$ and his team completed numerical simulation of free-burning argon arc in the first place and conducted spectral measurement. With the deepening of the research on arc, Li Hoping ${ }^{[3]}$ and his team from Tsinghua University studied the influence of the sheath on temperature field, while Ambles ${ }^{[8]}$ and D. Bernard ${ }^{[9]}$ researched the influence of external magnetic field on free-burning arc. In addition, as listed in References ${ }^{[4-12]}$, Flag researched the electrode ablation in free-burning arc, but failed to analyze the influence of electrode ablation on arc flow field; in References ${ }^{[6-7]}$.

This paper presents the coupled solutions to the equation sets of flow field and electromagnetic field by using magneto hydrodynamic modelling based on magnetic vector potential and the redeveloped hydromechanics-oriented software FLUENT.

\section{Magneto hydrodynamic (MHD) Model}

The magneto hydrodynamic model based on magnetic vector potential is adopted in the paper. For basic assumption and governing equation, see Reference ${ }^{[11]}$.

In order to complete coupling calculation of momentum equation, the circumferential component $B_{\theta}$ of the self-induction magnetic field intensity shall be solved. The following integrals are adopted in References [1-3]:

$$
B_{\theta}=\frac{\mu_{0}}{\mathrm{r}} \int_{0}^{\mathrm{r}} \mathrm{j}_{\mathrm{x}} \xi \mathrm{d} \xi
$$

The complicated integral calculation can be effectively avoided by expressing the magnetic field intensity in the magnetic vector potential model in the following form:

$$
\mathbf{B}=\nabla \times \mathbf{A}
$$

Ampere's law $\nabla \times \mathbf{B}=\mu_{0} \mathbf{J}$ can be rewritten into Poisson equation in the following form through Formula (2): $-\nabla^{2} \square \mathbf{A}=\mu_{0} \boldsymbol{J}$

Where $\mu_{0}$ is the vacuum magnetic permeability $\left(4 \pi \times 10^{-7} \mathrm{H} / \mathrm{m}\right)$. The following can be obtained by projecting (3) on the coordinate axis:

$$
\frac{1}{\mathrm{r}} \frac{\partial}{\partial \mathrm{r}}\left(\mathrm{r} \frac{\partial A_{\mathrm{x}}}{\partial \mathrm{r}}\right)+\frac{\partial}{\partial \mathrm{x}}\left(\frac{\partial A_{\mathrm{x}}}{\partial \mathrm{x}}\right)=-\mu_{0} \mathrm{j}_{\mathrm{x}}
$$




$$
\frac{1}{\mathrm{r}} \frac{\partial}{\partial \mathrm{r}}\left(\mathrm{r} \frac{\partial \mathrm{A}_{\mathrm{r}}}{\partial \mathrm{r}}\right)+\frac{\partial}{\partial \mathrm{x}}\left(\frac{\partial A_{\mathrm{r}}}{\partial \mathrm{x}}\right)=-\mu_{0} \mathrm{j}_{\mathrm{r}}+\frac{A_{\mathrm{F}}}{\mathrm{r}^{2}}
$$

Equations (4) and (5) are the scalar equations of magnetic vector potential. After the magnetic vector potential is calculated from two formulas, the magnetic field intensity can be directly calculated through (2). Under the 2-dimensional axially symmetric coordinates, the magnetic field intensity is:

$$
B_{\theta}=\frac{\partial A_{r}}{\partial \mathrm{x}}-\frac{\partial A_{\mathrm{x}}}{\partial \mathrm{r}}
$$

\section{Numerical Simulation of Plasma Arc based on FLUENT}

FLUENT is one of CFD software widely used in the world at present, which can simulate flow, heat transfer, combustion and other processes. In recent years, overseas scholars have applied the redeveloped FLUENT in the research of plasma arc. Murphy ${ }^{[10]}$ gives the thermodynamic properties and transport coefficients of commonly used gas plasma within a wide temperature range through theoretical calculation. Data in Reference ${ }^{[10]}$ is quoted in the paper.

\section{Boundary conditions}

The computational domain aimed at analysis and calculation in the paper is as shown in Figure 1, and boundary conditions are listed in Table 1. Axial symmetry boundary conditions are used on the center line $\mathrm{AB}$. On $\mathrm{BC}$ plane, no-slip boundary conditions are adopted, and the electrical potential is constant. There is no electric current passing through the outflow $\mathrm{CD}$, and the gas flow rate is maintained balanced with that of the inflow.

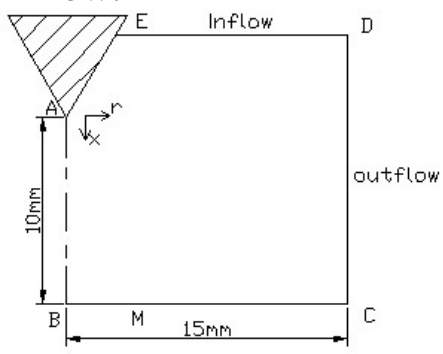

Fig. 1 Schematic diagram of free-burning arc gear

Table 1 MHD model boundary conditions

\begin{tabular}{clllll}
\hline & $\mathrm{AB}$ & $\mathrm{BC}$ & $\mathrm{CD}$ & $\mathrm{DE}$ & $\mathrm{EA}$ \\
\hline $\mathrm{u}$ & $\frac{\partial u}{\partial r}=0$ & $\mathrm{u}=0$ & $\frac{\partial u}{\partial r}=0$ & $\frac{\partial \rho u}{\partial r}=0$ & $\mathrm{u}=0$ \\
$\mathrm{v}$ & $\mathrm{v}=0$ & $\mathrm{v}=0$ & $\frac{\partial v}{\partial r}=C$ & $\mathrm{v}=0$ & $\mathrm{v}=0$ \\
$\mathrm{~T}$ & $\frac{\partial \mathrm{T}}{\partial r}=0$ & $1000 \mathrm{~K}$ & $300 \mathrm{~K}$ & $300 \mathrm{~K}$ & $3000 \mathrm{~K}$ \\
$\phi$ & $\frac{\partial \varphi}{\partial r}=0$ & $\varphi=0$ & $\frac{\partial \varphi}{\partial r}=0$ & $\frac{\partial \varphi}{\partial r}=0$ & Formula (7) \\
$A_{x}$ & $\frac{\partial \mathrm{A}_{\mathrm{x}}}{\partial r}=0$ & $\frac{\partial \mathrm{A}_{\mathrm{x}}}{\partial r}=0$ & 0 & $\frac{\partial A_{\mathrm{x}}}{\partial r}=0$ & $\frac{\partial A_{\mathrm{x}}}{\partial r}=0$ \\
$A_{r}$ & $\frac{\partial \mathrm{A}_{r}}{\partial r}=0$ & $\frac{\partial \mathrm{A}_{r}}{\partial r}=0$ & 0 & $\frac{\partial A_{r}}{\partial r}=0$ & $\frac{\partial A_{r}}{\partial r}=0$ \\
\hline
\end{tabular}

Discussion on the rationality of boundary conditions and initialization settings of the current continuity equation. In the setting of the whole MHD boundary conditions, the most important is the current density on cathode AE surface. According to Richardson - Dushman formula $\left(\mathrm{j}=A T^{2} e^{-\varphi / k_{\mathrm{B}} T}\right)$, thermionic emission completely depends on temperature. In the formula, $\mathrm{A}$ is the constant, and $\varphi_{\mathrm{c}}$ is the work function. There is a certain difficulty in directly calculating $j_{\max }$ through Richardson Dushman formula, because the work function of cathode surface material and its actual temperature 
cannot be measured. In order to avoid treatment of the cathode sheath and meanwhile meet the current continuity law, the current density distribution on the cathode surface is directly assumed. The current density is shown as follows:

$$
\left\{\begin{array}{l}
j_{c}=I / 2 \pi R_{c}^{2}, r \leq R_{c} \\
j_{c}=0, r>R_{c}
\end{array}\right.
$$

According to experimental observation of arc in Reference ${ }^{[1]}$, the radius of arc root of $200 \mathrm{~A}$ free-burning arc is about $0.51 \mathrm{~mm}$ ("white hot").

\section{Calculation Results and Discussion}

Comparison and analysis. Figure 2 shows comparisons between the isothermal chart obtained from calculation of arc temperature field and that generated by measured value from Hsu's experiments in $100 \mathrm{~A}$ and 200A respectively. In the arc center, the calculation results in the paper match Hsu's experiment results. However, at the arc edge, the local thermodynamic equilibrium and other assumptions are not satisfied due to temperature reduction, creating a deviation between calculation and experiment values. It is observed that the maximum temperatures of arc appear near the cathode tip. In the case of $100 \mathrm{~A}$, the maximum temperature is about $17,000 \mathrm{~K}$; in $200 \mathrm{~A}$, the maximum temperature is around $21,000 \mathrm{~K}$. After it is energized, the current gathers at the cathode tip, and punctures the surrounding air. With the action of joule heat, conductive thermal plasma is generated.
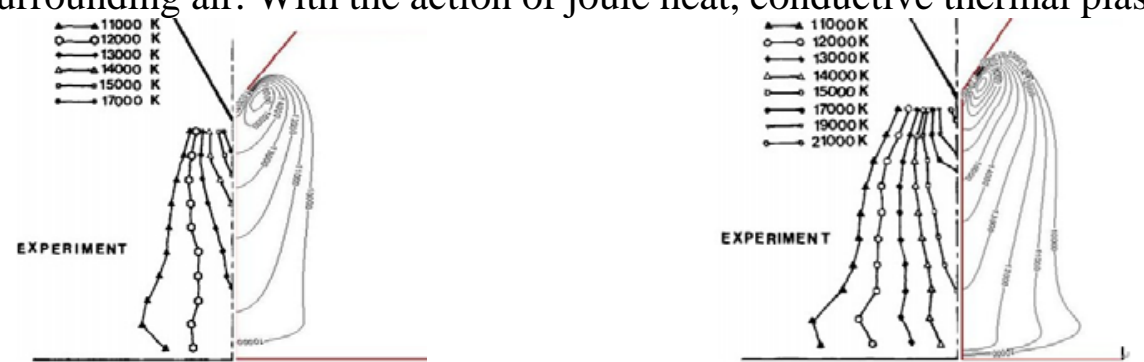

Fig. 2 Isothermal chart of $100 \mathrm{~A}$ and $200 \mathrm{~A}$ free-burning arc

In Figure 3, the axial velocity and pressure in 200A free-burning arc are compared. It is clearly seen that axial velocity drastically increases around the cathode tip due to the large pressure gradient caused by Lorentz force and generates cathode jet; after the plasma moves to the anode surface, it flows to the outlet along the anode sheet due to stagnation, and the axial velocity of plasma rapidly decreases to 0 , while the pressure rises as a result of compression.

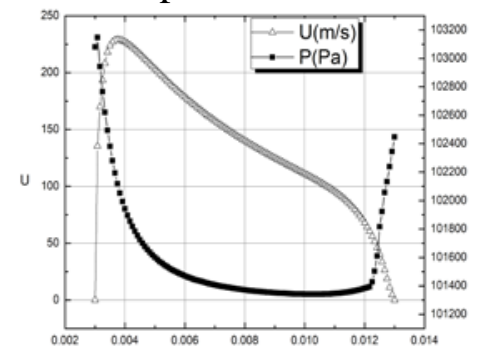

Figure 3 Axial velocity and pressure distribution of free-burning arc

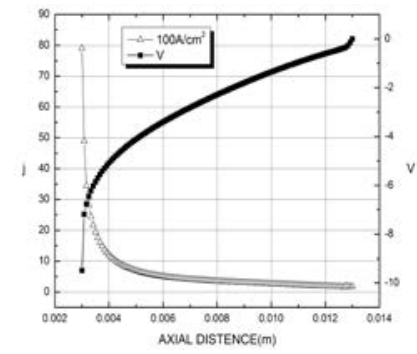

Fig.4 Axial current and voltage distribution of free-burning arc

Figure 4 indicates the axial distribution of 200A arc current and voltage. When it is just electrified, the cold air does not conduct electricity basically, and the current gathers at the cathode tip continuously. The current density at the cathode tip is obviously very high and punctures the air in the end.

\section{Influence of cathode deformation}

At high temperature, the sharp end of the cathode tip melts into a blunt-nosed frustum. According to the observation results of Zhou et al., the cathode tip is set as a frustum with a radius of $0.1 \mathrm{~mm}$ in consideration of its influence on the arc. 
It can be seen from Figure 6 that after the cathode tip melts into a frustum, the temperature near the cathode axis is slightly reduced, and the temperature decline curve tends to be smooth. This can be attributed to the extremely high temperature near the cathode tip (above 10000K), which melts the cathode tip into a frustum and therefore enlarges the contact area of the cathode tip and the plasma jet.

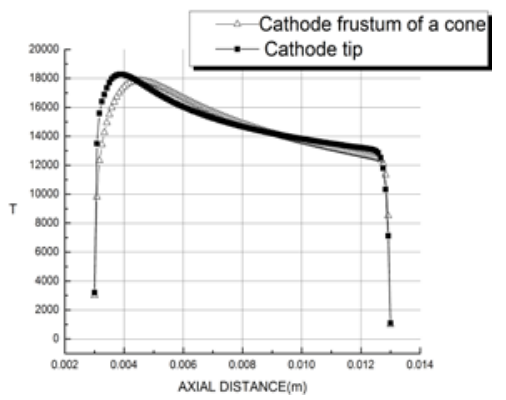

Fig. 5 A comparison of axis temperature between cathode frustum of a cone and cathode tip

\section{Conclusion}

Reasonable simplification of MHD equation sets based on magnetic vector potential can achieve rapid and accurate simulation of the plasma arc, and the calculation results match Hsu's experiment results presented in Reference ${ }^{[1]}$.

As to the treatment of boundary conditions for the electric current continuity equation, the arc root radius is restricted at the cathode tip, which can effectively reflect current concentration at the cathode tip. Reasonable boundary conditions and initialization settings enable a rapid and accurate simulation of the process where the plasma continuously accelerates the generation of cathode jet under the effect of axial Lorentz force, and finally the plasma arc comes into being. In the modified numerical model, the interaction of cathode tip melting, anode vapor and arc is brought into consideration. By means of calculation, the influence of these factors on the free-burning arc is analyzed, so that the calculation results can better reflect the actual situation.

\section{References}

[1] Hsu KC, Etemadi K, Pfender E. J Appl Phys,1983,53(3): p.1293-1301

[2] J.Menart,L.Lin, Numerical Study of a Free-Burning Argon Arc with Copper Contamination from the Anode.1998: p.153-170

[3] Xi Chen,He-Ping Li, an improved modeling approach.2000: p.2541-2553

[4] F.Lago, J.JGonzalez, P.Freton and A.Gleizes. J.Phys. D:Appl.Phys.,Vol 37, 2004, p. 883-897

[5] F.Lago,J.J.GonzaleZ, P.FretonandA.Gleizes. J.Phys. D:Appl.Phys.,Vol.38,2005: p.306-318

[6] R.Bini,M.Monno,M.1.Boulos. J.Phys.D:APPI.Phys,VOI,39,2006: p.3253-326

[7] R.Bini,M.Monno andM.1.Boulos. Plasma Chem. Plasma process, Vol. 27, 2007: p.359-380

[8] A.Blais,P.Proulx,M.1.Boulos. J.Phys.D:Appl.Phys ,Vol.36 2003: p.488-496

[9] D.Bernardi, V.Colombo, E.Ghelini, S.Melini, A.Mentralli, IEEE Trans. Plasma Sci. , Vol.33,2005, p.428-429

[10] Murphy A B. Plasma Process ., 1995,15: p.279-307

[11] Tian Guojun, Deng Jing, Li Yaojian, Journal of Theoretical and Applied Mechanics,2011,43: p.32-38

[12] Valerian Nemchinsky. Applied Physics,vol.45,2012,13: p.1-8. 